\title{
Perioperative mannitol intensive use may avoid the early complication of cerebral venous sinus stenting
}

\author{
Chaobo Bai ${ }^{1,2,3 \#}$, Jian Chen ${ }^{2,3,4 \#}$, Xiaoqin $\mathrm{Wu}^{1,2,3}$, Yuchuan Ding ${ }^{3,5}$, Xunming $\mathrm{Ji}^{2,3,4}$, Ran Meng ${ }^{1,2,3}$ \\ ${ }^{1}$ Department of Neurology, Xuanwu Hospital, Capital Medical University, Beijing, China; ${ }^{2}$ Advanced Center of Stroke, Beijing Institute for Brain \\ Disorders, Beijing, China; ${ }^{3}$ Department of China-America Institute of Neuroscience, ${ }^{4}$ Department of Neurosurgery, Xuanwu Hospital, Capital \\ Medical University, Beijing, China; ${ }^{5}$ Department of Neurosurgery, Wayne State University School of Medicine, Detroit, MI, USA \\ Contributions: (I) Conception and design: C Bai, J Chen, R Meng; (II) Administrative support: Y Ding, X Ji; (III) Provision of study materials or \\ patients: C Bai, J Chen; (IV) Collection and assembly of data: C Bai, J Chen, X Wu; (V) Data analysis and interpretation: C Bai; (VI) Manuscript \\ writing: All authors; (VII) Final approval of manuscript: All authors. \\ "These authors contributed equally to this work. \\ Correspondence to: Ran Meng, MD, PhD. Department of Neurology, Department of China-America Institute of Neuroscience, Xuanwu Hospital, \\ Capital Medical University, Beijing, China. Email: victor65@126.com.
}

Background: Cerebral venous sinus (CVS) stenting has been widely applied for correcting CVS stenosis. However, there are still some potential complications. The purpose of this study is to investigate the impact of perioperative management on avoiding complications of CVS stenting.

Methods: Patients confirmed as CVS stenosis were enrolled from January 2014 through November 2019. All CVS stenosis were corrected by stenting when the trans-stenotic mean pressure gradient (MPG) was up to or over $8 \mathrm{mmHg}$. Patients were divided into perioperative management group and control group. Patients in the former group underwent transiently mannitol $250 \mathrm{~mL}$ intravenous infusion immediately prior to stenting besides routine ICP control. While patients in control group underwent the same routine treatment as in the perioperative management group. The clinical symptoms, intracranial pressure (ICP), and MPG of the patients were compared before and after stenting. In addition, the complications between the two groups were compared.

Results: A total of 81 eligible patients were finally enrolled in this study, including 64 females and 17 males, mean aged $45.35 \pm 13.83$ years. After stenting, the stenotic CVS restored normal blood flow and MPG decreased significantly [10.0 (8.0-15.0) vs. $0.0(0.0-0.7) \mathrm{mmHg}, \mathrm{P}<0.001]$. Headache, tinnitus, visual impairment, visual loss, Frisén papilledema grade (FPG), and ICP were ameliorated immediately $(\mathrm{P}<0.001)$ in the majority of patients in the two groups. However, the incidence of intracranial hemorrhage was higher in control group $(11.4 \%$ vs. $0.0 \%, \mathrm{P}=0.031)$.

Conclusions: A transiently strict preoperative ICP control by mannitol may inhibit CVS stenting-related hemorrhage, which makes the stenting safer and more effective on correcting the CVS stenosis.

Keywords: Cerebral venous sinus stenosis (CVS stenosis); stenting; perioperative management; complication

Submitted Mar 31, 2020. Accepted for publication Apr 27, 2020.

doi: 10.21037/atm-20-3021

View this article at: http://dx.doi.org/10.21037/atm-20-3021

\section{Introduction}

Cerebral venous sinus (CVS) stenosis is closely related to intracranial hypertension (IH). CVS stenosis can induce cerebral venous outflow disorders, which subsequently result in intracranial pressure (ICP) elevation (1-3). Some scholars have proposed the hypothesis that the CVS stenosis and the elevated ICP may be mutually reinforcing (4). CVS stenosis can be manifest as severe headache and persistent tinnitus. In some patients, CVS stenosis represents visual impairment, which may lead to progressive vision loss if not corrected immediately. In recent years, the effectiveness of stenting has been well established in CVS stenosis (5-8). 
Venous stenting has advantages in the aspects of reducing $\mathrm{IH}$, alleviating clinical symptoms, and preventing or improving visual impairment.

Cerebral venous stenting has become a promising treatment option for CVS stenosis. However, the CVS stenting-related complications have not received enough attention. The common complications include postoperative headache, epidural hemorrhage, cerebral hemorrhage, and restenosis and intra-stent thrombosis. Serious complications such as life-threatening cerebral hemorrhage may lead to a poor clinical outcome (8-10). This study aimed to explore the efficacy of perioperative management on preventing CVS stenting-related complications.

We present the following article in accordance with the STROBE reporting checklist (available at http://dx.doi. org/10.21037/atm-20-3021).

\section{Methods}

\section{Study design and patient's selection}

This single center real-world study was approved by the Institutional Ethics Committee. All participants signed the consent forms before enrolment. The study was conducted from January 2014 through November 2019. All medical data from the inpatient database were obtained and analyzed by two experienced neurologists and radiologists. The clinical data such as clinical characteristics, ICP, and mean pressure gradient (MPG) before and after stenting, as well as the complications, were analyzed.

Patient inclusion criteria for initial stenting were: (I) patients with CVS stenosis confirmed by contrast-enhanced MR venography (CE-MRV)/CT venography (CTV)/digital subtraction angiography (DSA) were eligible for this study; (II) lumbar puncture opening pressure $\geq 200 \mathrm{mmH}_{2} \mathrm{O}$; (III) the trans-stenotic MPG $\geq 8 \mathrm{mmHg}$; (IV) patients were unresponsive to conservative medical treatment.

Exclusion criteria: (I) intracranial central nervous system (CNS) malignancy; (II) patient with incomplete clinical data or lack of the follow-up data.

\section{Treatment procedure}

\section{Preoperative preparation}

All patients preparing for undergoing stenting procedure received dual antiplatelet daily [75 $\mathrm{mg}$ clopidogrel (Sanofi Winthrop Industries, France, the approval number was J20130083) and $100 \mathrm{mg}$ of aspirin (Bayer Healthcare
Manufacturing, Italy, the approval number was J20171021)] for 3 to 5 days, besides routine dehydration treatment, such as mannitol (produced by Beijing Huarunshuanghe Pharmaceutical Co., Ltd., the approval number was H11020861) $125 \mathrm{~mL} / \mathrm{q} 6 \mathrm{~h} / \mathrm{iv}$. In addition, patients were divided into two groups according to whether or not they agreed to undergo the perioperative strict ICP control. Patients in perioperative management group underwent transiently mannitol $250 \mathrm{~mL}$ intravenous infusion immediately prior to stenting, whereas, patients in control group did not undergo transiently mannitol $250 \mathrm{~mL}$ intravenous infusion except for the same routine treatment as in the perioperative management group.

\section{CVS manometry and stenting}

Diagnostic cerebral sinus venography and manometry were performed under local anesthesia. All patients were heparinized prior to CVS manometry. Eligible patients (MPG $\geq 8 \mathrm{mmHg}$ ) were treated with self-expanding Acculink stenting (Cordis, Hialeah, Florida, USA).

\section{Postoperative management}

All patients in the two groups underwent low molecular weight heparin (Sanofi Winthrop Industries, France, the approval number was J20150059) subcutaneous injection $(0.6 \mathrm{~mL}, \mathrm{q} 12 \mathrm{~h})$ and conventional dehydration therapy before stenting for 3 days. Blood pressure, heart rate, and blood oxygen saturation of the patients were monitored. CT scan of the brain was routinely performed within $24 \mathrm{~h}$ post-stenting. If no evidence of intracranial hemorrhage in their CT maps, the patients would continue undergo dual antiplatelet for 12 months. In addition, patients with CVSTinduced CVS stenosis would undergo single antiplatelet and single oral anticoagulation agent for 6 months, after which single antiplatelet monotherapy was administered for 1 year. All cases were followed up by outpatient or telephone.

\section{Quantification of clinical characteristics}

The severity of papilledema was assessed by Frisén papilledema grade (FPG). ICP was defined as the lumbar puncture open pressure. The trans-stenotic pressure gradient was represented by MPG.

\section{Statistical analysis}

We performed all data analysis by the social science statistical software package SPSS version 21.0 program (IBM, USA). 
Table 1 Baseline demographic data

\begin{tabular}{|c|c|}
\hline Clinical characteristics & Items \\
\hline \multicolumn{2}{|l|}{ Personal data } \\
\hline Age & $45.35 \pm 13.83$ \\
\hline Gender (male/female) & $17 / 64$ \\
\hline Mean BMI (kg/m²) & $26.53 \pm 4.13$ \\
\hline Time from onset to door, month & $6[2-24]$ \\
\hline Mean follow up duration, month & $23.6(12.1-42.3)$ \\
\hline \multicolumn{2}{|l|}{ Clinical manifestations, $\mathrm{n}(\%)$} \\
\hline Headache & $38(46.9)$ \\
\hline Tinnitus & $26(32.1)$ \\
\hline Visual disorders & $33(40.7)$ \\
\hline Visual loss & $48(59.3)$ \\
\hline \multicolumn{2}{|l|}{ Comorbidities, n (\%) } \\
\hline CVST & $11(13.6)$ \\
\hline Type 2 diabetes mellitus & $4(4.9)$ \\
\hline Hypertension & $28(34.6)$ \\
\hline Hyperlipemia & $10(12.3)$ \\
\hline Heart disease & $4(4.9)$ \\
\hline \multicolumn{2}{|l|}{ Lifestyle habits, n (\%) } \\
\hline Smoking & $6(7.4)$ \\
\hline Alcohol drinking & $5(6.2)$ \\
\hline \multicolumn{2}{|l|}{ Side of CVS stenosis, $\mathrm{n}(\%)$} \\
\hline Left TS & $33(40.7)$ \\
\hline Right TS & $36(44.4)$ \\
\hline Superior sagittal sinus & $4(4.9)$ \\
\hline Straight sinus & $1(1.2)$ \\
\hline Left TS-SS boundary & $32(39.5)$ \\
\hline Right TS-SS boundary & $42(51.9)$ \\
\hline
\end{tabular}

BMI, body mass index; CVST, cerebral venous sinus thrombosis; TS, transverse sinus; SS, sigmoid sinus.

The results were expressed as counts and percentages for categorical variables, as mean \pm standard deviation for continuous variables, and as median (interquartile range) for discrete data. The Fisher's exact test was applied to evaluate differences in categorical variables, and the Student $t$-test to evaluate differences in continuous variables. For data that did not meet the normal distribution, Mann-Whitney $\mathrm{U}$ test was applied. Two-sided $\mathrm{P}$ values $<0.05$ were defined as statistical significance for all tests.

\section{Results}

\section{Baseline demographic data}

The baseline demographic data were shown in Table 1. In total, 81 patients met the inclusion criteria, the mean age was $45.35 \pm 13.83$ years, and female vs. male was $64 v s .17$. The mean BMI was $26.53 \pm 4.13 \mathrm{~kg} / \mathrm{m}^{2}$. The median time from symptoms onset to door was 6 [2-24] months. The average time of follow-up was $23.6(12.1-42.3)$ months. Common clinical manifestations of CVS stenosis were visual loss $(59.3 \%)$, headache $(46.9 \%)$, visual disorders $(40.7 \%)$ and tinnitus (32.1\%). For lifestyle habits, in which $7.4 \%$ reported smoking and $6.2 \%$ had alcohol abuse. For the positions of CVS stenosis, transverse sinus (left accounted for $40.7 \%$ and right was $44.4 \%$ ) and transverse sinus-sigmoid sinus boundary (left accounted for $39.5 \%$ and right was $51.9 \%$ ) were the top two vulnerable segments. In addition, superior sagittal sinus $(4.9 \%)$ and straight sinus $(1.2 \%)$ were also affected. Finally, 46 patients in the perioperative management group and 35 patients in control group were analyzed and compared.

\section{Comparison of clinical data before and after stenting}

The clinical characteristics of the patients with CVS stenosis prior and post-stenting were shown in Table 2. Symptoms such as headache, visual disorders, visual loss and tinnitus significantly improved after stenting $(\mathrm{P}<0.001)$. Although the number of patients with papilledema did not attenuate significantly at a few initial days post-stenting, the FPG of the papilledema at post-stenting was significantly ameliorated than that prior to stenting [pre- $v s$. post-stenting $=3.0(3.0-4.0) v s$. 1.0 (1.0-1.5), $\mathrm{P}<0.001]$. In addition, significant reduction in ICP was observed (pre- $v s$. post-stenting $=296.84 \pm 64.52 v s$. $\left.199.74 \pm 56.43 \mathrm{mmH}_{2} \mathrm{O}, \mathrm{P}<0.001\right)$. The MPG pre- and poststenting was measured in situ during catheter angiography. Comparing the MPG pre- and post-stenting, the abnormally elevated MPG decreased immediately and remarkably when the CVS stenosis was corrected by stenting [10.0 (8.0-15.0) vs. $0.0(0.0-0.7) \mathrm{mmHg}, \mathrm{P}<0.001]$.

\section{Comparison of clinical data between perioperative group and control group}

The clinical characteristics of the patients in perioperative management group and control group were displayed in Table 3. There were no significant differences in clinical symptoms, FPG, ICP, and MPG between the two groups 
Table 2 Clinical characteristics of patients with CVS stenosis

\begin{tabular}{lccc}
\hline Items & Pre-stenting & Post-stenting & P value \\
\hline Symptoms, $\mathrm{n}(\%)$ & & 0 & $<$ \\
Headache & $38(46.9)$ & 0 & $<0.001$ \\
Tinnitus & $26(32.1)$ & $3(3.7)$ & $1(1.2)$ \\
Visual impairment & $33(40.7)$ & $49(60.5)$ & $<0.001$ \\
Visual loss & $33(40.7)$ & $1.0(1.0-1.5)$ & $<0.001$ \\
Papilledema & $53(65.4)$ & $199.74 \pm 56.43$ & $<0.626$ \\
FPG & $3.0(3.0-4.0)$ & $0.0(0.0-0.7)$ & $<0.001$
\end{tabular}

CVS, cerebral venous sinus; FPG, Frisén papilledema grade; ICP, intracranial pressure; MPG, mean pressure gradient.

Table 3 Clinical characteristics of perioperative management group and control

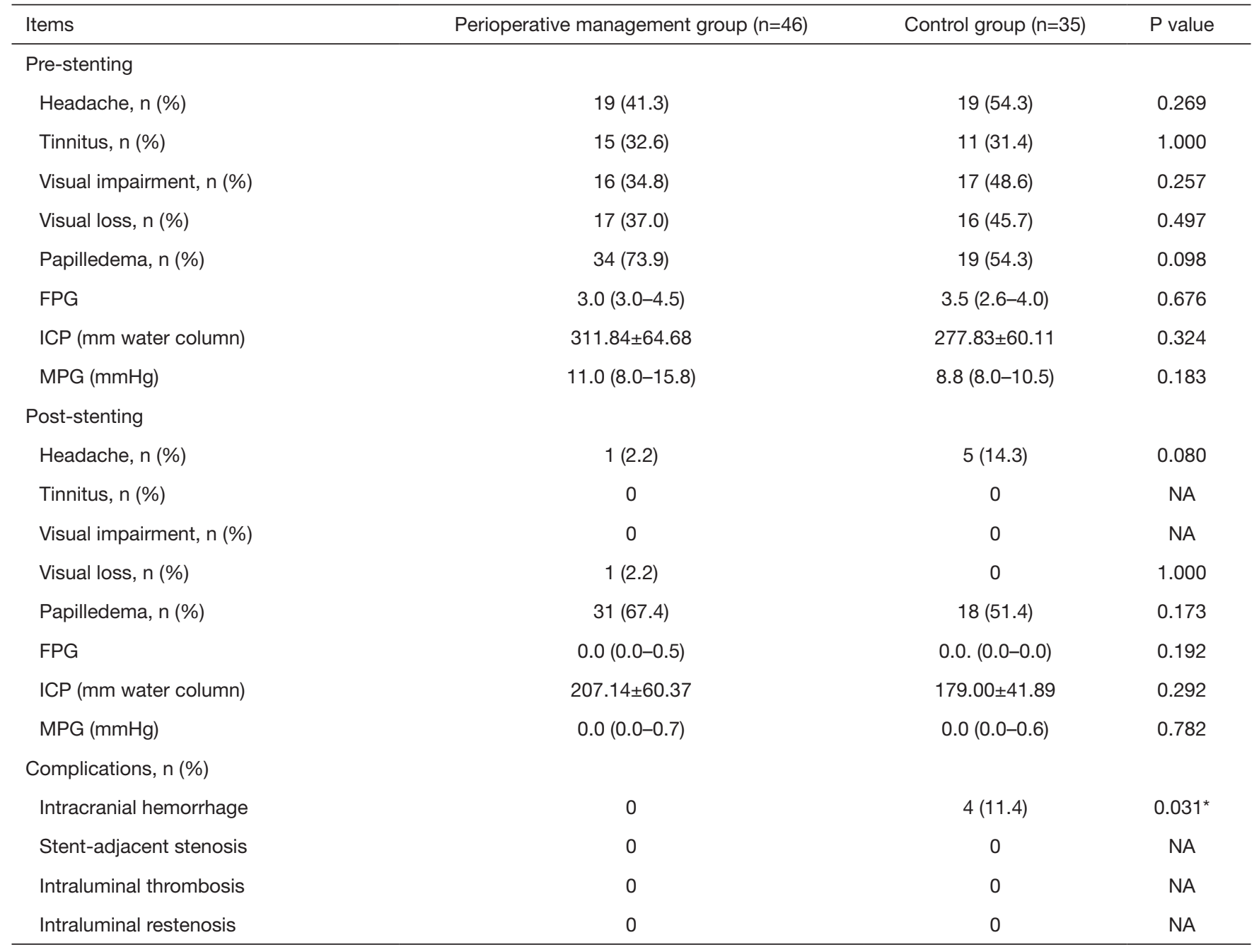

Data are presented as $\mathrm{n}(\%)$, mean $\pm \mathrm{SD}$ or median (interquartile range). ${ }^{*}, \mathrm{P}<0.05$. FPG, Frisén papilledema grade; ICP, intracranial pressure; MPG, mean pressure gradient. 

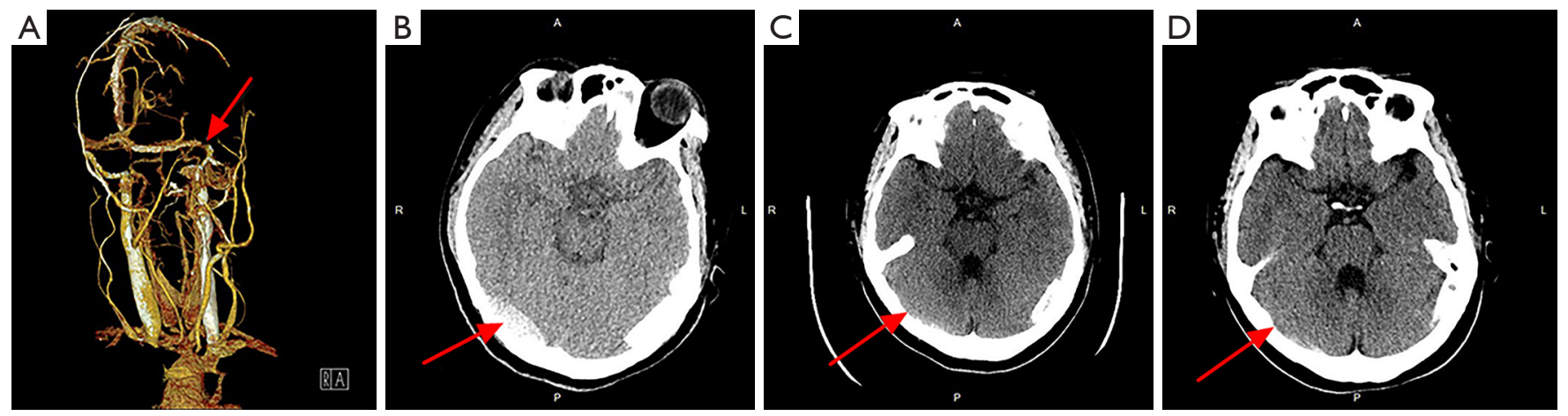

Figure 1 Image of a mild epidural hemorrhage in a patient after CVS-stenting. The initial CTV revealed stenosis at the border of the left transverse sinus and sigmoid sinus (A, red arrow); however, the patient presented with severe headache 3 h post-stenting, and CT followup showed left epidural hemorrhage (B, red arrow); the symptoms gradually eased after aggressive dehydration therapy, and another two followed-up CT maps at day-5 (C, red arrow) and day-8 (D, red arrow) after CVS-stenting revealed complete absorption of the epidural hemorrhage. CVS, cerebral venous sinus; CTV, CT venography.

at baseline. However, the ratio of headache post-stenting in the perioperative management group was much lower than that in control group ( $2.2 \%$ vs. $14.3 \%, \mathrm{P}=0.080)$. Other items, such as clinical symptoms, FPG, ICP and MPG showed no significant difference between the two groups.

For early complications post-stenting, 4 patients (11.4\%) in control group complicated with stenting-related subdural hematoma, all the hematomas located at the contralateral of the stenting side (Figures 1,2). Whereas, no subdural hematoma event $(0.0 \%)$ occurred in perioperative management group, $\mathrm{P}=0.031$. Other complications, such as stent-adjacent stenosis, intraluminal thrombosis, and intraluminal restenosis showed no statistic difference between the two groups.

\section{Discussion}

\section{CVS stenosis and idiopathic intracranial bypertension (IIH)}

Previous studies found that IIH involved more common in obese women and was poor response to medication treatment $(3,4)$. However, increasing studies in recent years revealed that CVS stenosis might be one of the major etiologies of refractory IIH, and further, it is worth noting that cerebral sinus stenting can significantly reduce ICP and obtain relief of clinical symptoms accordingly (11-13). Therefore, CVS stenosis received increasing attention as a potential cause of $\mathrm{IH}(7,14)$.

\section{Clinical and imaging characteristic of CVS stenosis}

In our study, IH-related headaches and visual loss were prominent clinical symptoms of CVS stenosis. Notably, the degree of headache varies from case to case. However, chronic, persistent and tolerable headaches may be missed diagnosis $(7,15,16)$. Long-term optic papillary edema caused by IH led to ischemia of the optic disc, and then resulted in visual damage. In addition, pulsatile tinnitus is another common symptom of CVS stenosis. Although the pathological mechanism of pulsatile tinnitus has not been elucidated, its response to stenting surgery is satisfactory (17-19). To date, a variety of methods including MRV, CTV, and DSA have been applied to detect CVS stenosis. In our study, transverse sinus and transverse sinus-sigmoid sinus boundary are the most common affected sites.

\section{Clinical CVS stenting-related hemorrbage}

The operating environment is different between CVS stenting and cerebral arterial stenting. The former is performed under IH, while the later was not. When the CVS outflow was opened suddenly by stenting, a transient pressure difference between the side of the stent and the contralateral brain may occur, resulting in midline shift, the contralateral perforating vein is pulled and damaged, as a result, the risk of intracranial hemorrhage and cerebral hematoma may increase. Whereby, potential CVS stenting-related early complications involved intracranial hemorrhage and subdural hematoma $(14,20)$. Thus, the intensive ICP control may attenuate the pressure difference between the two sides of the midline immediately after stenting. This may explain the results in this study that the 

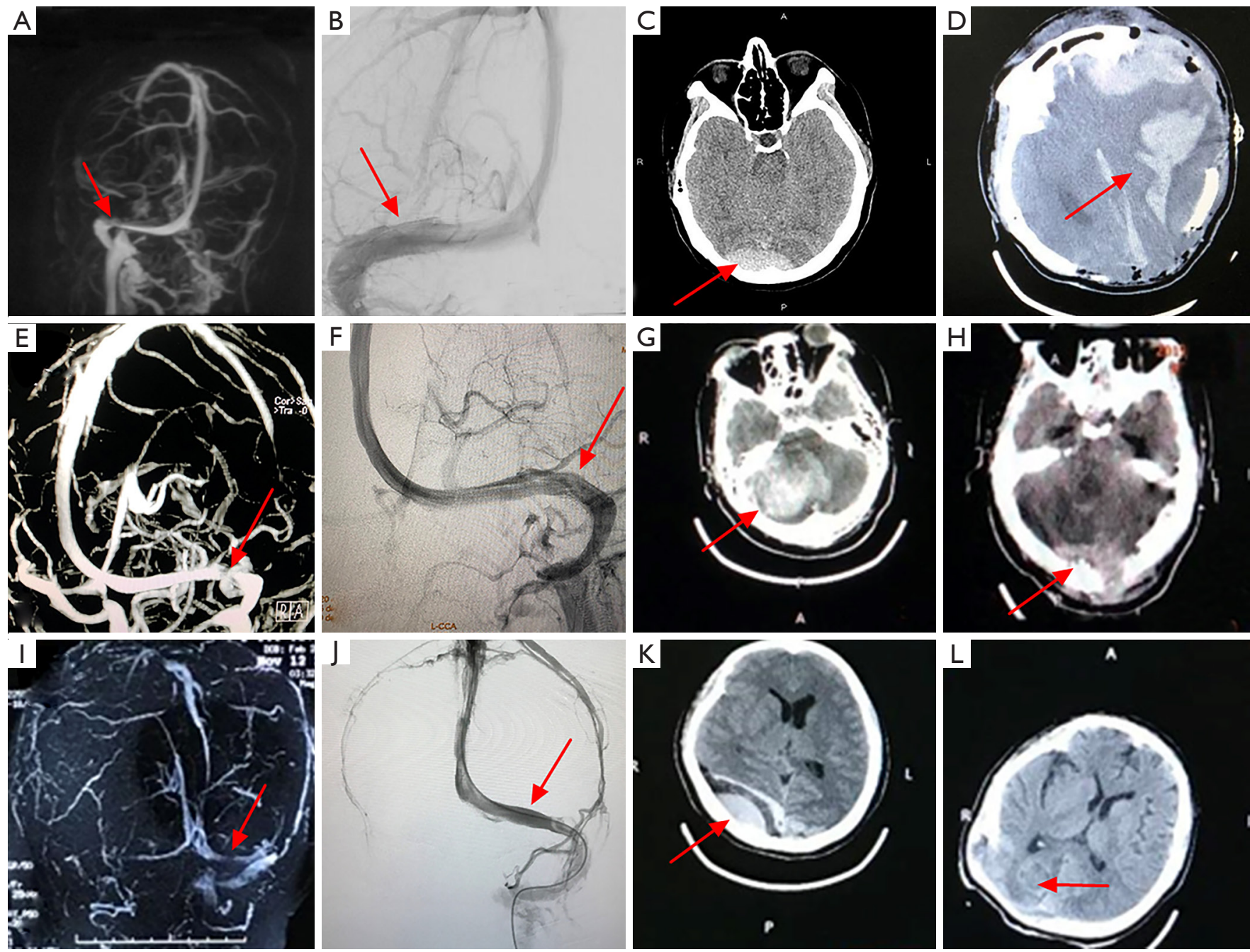

Figure 2 Images of severe intracranial hemorrhage after stenting in three patients (patient 1: A-D; patient 2: E-H; patient 3: I-L). Their initial MRV and DSA revealed CVS stenosis (A,B,E,F,I,J, red arrow); their symptoms and signs once improved transiently after stenting. However, they showed severe headaches, vomiting and decreased levels of consciousness, which progressed to a coma after stenting. CT scan revealed intracranial hemorrhage and swelling of the brain tissue (C, G,K, red arrow); they all immediately underwent life-saving decompressive craniectomy, and postoperative CT revealed severe intracranial hemorrhage (D,H,L, red arrow). However, our long-term follow-up found that they were still unconscious and rely on life support equipment. MRV, MR venography; DSA, digital subtraction angiography; CVS, cerebral venous sinus.

incidence of CVS stenting-related hematoma is lower in the perioperative management group than that in control group.

In addition, long-term complications of stenting include intraluminal thrombosis and intraluminal restenosis, the incidences of CVS stenting-related complications differed among previous literatures, ranged from $2 \%$ to $10 \%(14,20)$. For long-term complications such as stent-adjacent stenosis, intraluminal thrombosis and intraluminal restenosis, uncontrolled intimal proliferation may be the potential cause (20). Previous studies compared the effects of dual antiplatelet and single antiplatelet drugs on long-term complications after CVS stenting, all of which revealed that patients with dual antiplatelet therapy had a lower incidence of restenosis and thrombosis after the stent placement $(14,20,21)$. However, the long-term complications mentioned above did not occur in all of the patients enrolled in this study, 
which revealed that, both dual antiplatelet or antiplatelet combined with single anticoagulation may be a promising therapy to inhibit long-term complications after CVS stenting.

\section{Importance of strictly preoperative ICP control}

In this study, 4 out of 35 patients in control group (11.4\%) complicated with subdural hematoma, moreover, all of the hematomas located at the opposite side of the stent. However, no hematoma event occurred in the perioperative management group. The mechanism of the stenting-related contralateral side subdural hematoma was not clarified. A possible explanation for this phenomenon was that patients in the control group did not undergo a transiently strictly preoperative ICP reducing therapy. Therefore, we hypothesized that ICP on the stenting side was suddenly reduced after the stent self-expended, which resulted in a rapid pressure gradient of the ICP between two sides of the midline. The brain tissue on the opposite hemisphere may "drift" towards the stenting side to some degree. Whereby, a tear between the vessels in brain tissue and the dura mater resulted in a subdural hematoma. The same type of intracranial hemorrhage has also appeared on the side contralateral to the stenting in other studies $(5,22)$. Our results suggest that patients with unilateral transverse sinus stenosis and severe IH may benefit from transiently strictly ICP control. However, this hypothesis still needs more evidence to prove.

\section{Limitations and generalizability}

This study has several limitations. Firstly, this study is a single-center study, which may limit the number of patients we enrolled. Future multi-center research is urgently needed. Secondly, the mechanism of the impact of perioperative management on stenting is unclear. Our hypothesis needs more evidence and clinical trials to further confirm. Finally, our follow-up duration is not long enough, which may affect our conclusions on clinical outcomes. Our follow-up study is still ongoing.

As the operating environment of the intracranial venous stenting is differing from the intracranial artery stenting, and the former is usually in an IH environment. Mannitol intensive use decreases the IH prior to stenting, which can shrink the pressure difference between two sides of the midline after stenting. Thus, a transient strict ICP control during the perioperative period may be reasonable for inhibiting CVS stenting-related hemorrhagic complications.

\section{Conclusions}

A transiently strict preoperative ICP control by mannitol may inhibit CVS stenting-related hemorrhage, which makes the stenting safer and more effective on correcting the CVS stenosis.

\section{Acknowledgments}

We would like to thank all patients and doctors who participated in this study for their cooperation.

Funding: This study was sponsored by the National Key R\&D Program of China (2017YFC1308401), the National Natural Science Foundation (81371289), the Project of Beijing Municipal Top Talent for Healthy Work of China (2014-2-015).

\section{Footnote}

Reporting Checklist: The authors have completed the STROBE reporting checklist. Available at http://dx.doi. org/10.21037/atm-20-3021

Conflicts of Interest: All authors have completed the ICMJE uniform disclosure form (available at http://dx.doi. org/10.21037/atm-20-3021). The authors have no conflicts of interest to declare.

Ethical Statement: The authors are accountable for all aspects of the work in ensuring that questions related to the accuracy or integrity of any part of the work are appropriately investigated and resolved. This study was approved by the Ethics Committee of Xuanwu Hospital, Capital Medical University (No. 2019-006). All participants signed the consent forms before enrolment.

Open Access Statement: This is an Open Access article distributed in accordance with the Creative Commons Attribution-NonCommercial-NoDerivs 4.0 International License (CC BY-NC-ND 4.0), which permits the noncommercial replication and distribution of the article with the strict proviso that no changes or edits are made and the original work is properly cited (including links to both the formal publication through the relevant DOI and the license). See: https://creativecommons.org/licenses/by-nc-nd/4.0/. 


\section{References}

1. Bono F, Messina D, Giliberto C, et al. Bilateral transverse sinus stenosis predicts IIH without papilledema in patients with migraine. Neurology 2006;67:419-23.

2. Carvalho GB, Matas SL, Idagawa MH, et al. A new index for the assessment of transverse sinus stenosis for diagnosing idiopathic intracranial hypertension. J Neurointerv Surg 2017;9:173-7.

3. Riggeal BD, Bruce BB, Saindane AM, et al. Clinical course of idiopathic intracranial hypertension with transverse sinus stenosis. Neurology 2013;80:289-95.

4. Peng KP, Fuh JL, Wang SJ. High-pressure headaches: idiopathic intracranial hypertension and its mimics. Nat Rev Neurol 2012;8:700-10.

5. Ahmed RM, Wilkinson M, Parker GD, et al. Transverse sinus stenting for idiopathic intracranial hypertension: a review of 52 patients and of model predictions. AJNR Am J Neuroradiol 2011;32:1408-14.

6. Radvany MG, Solomon D, Nijjar S, et al. Visual and neurological outcomes following endovascular stenting for pseudotumor cerebri associated with transverse sinus stenosis. J Neuroophthalmol 2013;33:117-22.

7. Cappuzzo JM, Hess RM, Morrison JF, et al. Transverse venous stenting for the treatment of idiopathic intracranial hypertension, or pseudotumor cerebri. Neurosurg Focus 2018;45:E11.

8. Koovor JM, Lopez GV, Riley K, et al. Transverse venous sinus stenting for idiopathic intracranial hypertension: safety and feasibility. Neuroradiol J 2018;31:513-7.

9. Satti SR, Leishangthem L, Chaudry MI. Meta-analysis of CSF diversion procedures and dural venous sinus stenting in the setting of medically refractory idiopathic intracranial hypertension. AJNR Am J Neuroradiol 2015;36:1899-904.

10. Kumpe DA, Seinfeld J, Huang X, et al. Dural sinus stenting for idiopathic intracranial hypertension: factors associated with hemodynamic failure and management with extended stenting. J Neurointerv Surg 2017;9:867-74.

11. Bai C, Xu Y, Zhou D, et al. The comparative analysis of non-thrombotic internal jugular vein stenosis and cerebral venous sinus stenosis. J Thromb Thrombolysis 2019;48:61-7.

Cite this article as: Bai C, Chen J, Wu X, Ding Y, Ji X, Meng R. Perioperative mannitol intensive use may avoid the early complication of cerebral venous sinus stenting. Ann Transl Med 2020;8(11):672. doi: 10.21037/atm-20-3021
12. Xu Y, Meng R, Rajah GB, et al. Long-term outcomes of cerebral venous sinus stenosis corrected by stenting. Curr Neurovasc Res 2019;16:77-81.

13. Li K, Ren M, Meng R, et al. Efficacy of stenting in patients with cerebral venous sinus thrombosis-related cerebral venous sinus stenosis. J Neurointerv Surg 2019;11:307-12.

14. Fargen KM, Liu K, Garner RM, et al. Recommendations for the selection and treatment of patients with idiopathic intracranial hypertension for venous sinus stenting. J Neurointerv Surg 2018;10:1203-8.

15. Lenck S, Vallée F, Civelli V, et al. Assessment of blood flow velocities and venous pressures using a dualsensor guidewire in symptomatic dural sinus stenoses. J Neurosurg 2018. [Epub ahead of print].

16. Puffer RC, Mustafa W, Lanzino G. Venous sinus stenting for idiopathic intracranial hypertension: a review of the literature. J Neurointerv Surg 2013;5:483-6.

17. Baomin L, Yongbing S, Xiangyu C. Angioplasty and stenting for intractable pulsatile tinnitus caused by dural venous sinus stenosis: a case series report. Otol Neurotol 2014;35:366-70.

18. Lenck S, Labeyrie MA, Vallee F, et al. Stent placement for disabling pulsatile tinnitus caused by a lateral sinus stenosis: a retrospective study. Oper Neurosurg (Hagerstown) 2017;13:560-5.

19. Yang IH, Pereira VM, Lenck S, et al. Endovascular treatment of debilitating tinnitus secondary to cerebral venous sinus abnormalities: a literature review and technical illustration. J Neurointerv Surg 2019;11:841-6.

20. Saber H, Lewis W, Sadeghi M, et al. Stent survival and stent-adjacent stenosis rates following venous sinus stenting for idiopathic intracranial hypertension: a systematic review and meta-analysis. Interv Neurol 2018;7:490-500.

21. Higgins JN, Cousins C, Owler BK, et al. Idiopathic intracranial hypertension: 12 cases treated by venous sinus stenting. J Neurol Neurosurg Psychiatry 2003;74:1662-6.

22. Leishangthem L, SirDeshpande P, Dua D, et al. Dural venous sinus stenting for idiopathic intracranial hypertension: An updated review. J Neuroradiol 2019;46:148-54. 